\title{
Analysis of positive feedback in the control of movement
}

\author{
Soroor Behbahani ${ }^{1}$, Amir Homayoun Jafari $^{2}$ \\ ${ }^{1,2}$ Biomedical Engineering Department, Science and Research Branch, Islamic Azad University, Tehran, Iran. \\ Email: ${ }^{\text {soroor_behbahani@yahoo.com; }{ }^{2} \text { Amir_h_jafari@rcstim.ir }}$
}

Received 1 June 2009; revised 13 July 2009; accepted 15 July 2009.

\begin{abstract}
Over the past three decades, neurophysiologists studying the neural circuitry responsible for control of skeletal muscles have developed several different general theories of sensorimotor control. These have usually invoked one or more of the sources of proprioceptive signals (e.g. muscle spindle and Golgi tendon organ afferents) in positive or negative feedback loops to the homonymous alpha motoneurones. In this paper we consider to analyze the role of positive feedback in combination of negative feedback due to important role of them in stabilizing the neuromuscular system.
\end{abstract}

Keywords: Positive Feedback; Negative Feedback; Hill Model; Reflex Model

\section{INTRODUCTION}

The stretch reflex differs in decerebrate and intact animals and since Sherrington's time it has come to be realized that several CNS mechanisms may contribute components of different latency to the stretch reflex response. At the segmental level, muscle spindle Ia afferents activated by muscle lengthening monosynaptically excite homonymous alpha motoneurons which in turn cause the muscle to resist the stretch. In static postures Ib input generally results in homonymous inhibition, but it has been shown that this switches to longer-latency homonymous excitation during locomotion [1], at least in cat extensor muscles. Group II input from muscle spindles has also been implicated in long latency components of stretch reflexes [2,3,4]. Ia homonymous excitation represents negative displacement feedback, which augments the intrinsic stiffness of active muscles in the face of length perturbations. Ib homonymous feedback on the other hand represents positive force feedback. Positive feedback is synonymous with instability and oscillation in engineering systems, but when muscles are the actuators; their nonlinear lengthtension properties turn out to stabilize the positive feedback loop [5].

\section{INTERACTIONS BETWEEN POSITIVE AND NEGATIVEFEEDBACK}

According to linear systems theory, positive feedback loops alone produce instability, while negative loops alone may require very high gains to be useful. The general advantage of combining positive and negative feedback loops is that it reduces the sensitivity of system response to unpredictable variations in any of its components, for example, fatiguing of a muscle or increase of a mechanical load [5], without requiring excessive negative feedback gains.

On the other hand, Prochazka [6] demonstrated that positive force feedback could also perform this role assisted by negative length feedback.

Positive force feedback through group Ib afferents may work in parallel to the positive length feedback through the loop and/or group II afferent modulation of activity $[7,8]$. In the case of Ib force feedback, the loop is polysynaptic so that transmission through it is state dependent. One possible advantage of loops is that they influence and motoneurons monosynaptically [6].

In current research we analyzed the role of positive feedback in combination of negative feedback due to important role of them in stabilizing the neuromuscular system. We used a simple reflex model to show the behaviors of neuromuscular system. Whole of these models were done using MATLAB software.

\subsection{Reflex Model}

Figure 1 shows a highly simplified model of a reflex system. In this model, key elements of real neuromuscular systems such as tendon compliance, dynamic transfer functions of sensor, and length and velocity dependence of muscle force production are omitted or simplified. The load in this model is a mass of $1 \mathrm{~kg}$.

In this model, muscle force is modeled by a first-order active component and a viscoelastic parallel stiffness. The force velocity relationship [9,10], the muscle length tension curve and tendon compliance are all neglected. 12 are used to represent the image obtained by limb leads. 


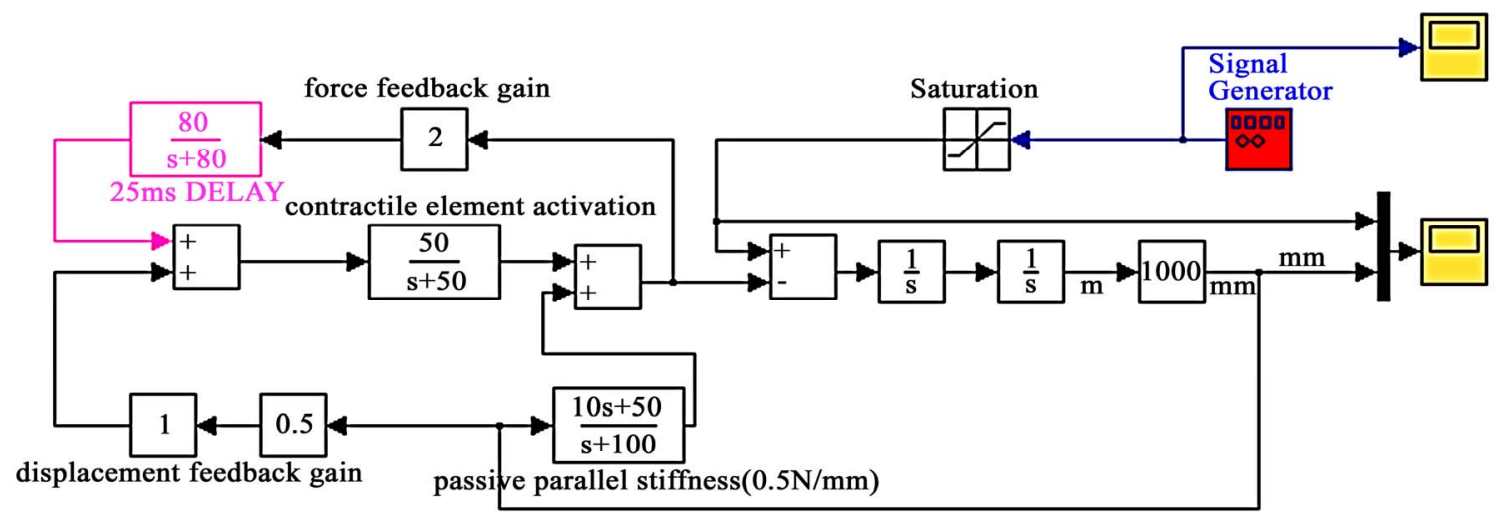

Figure 1. Reflex model.

The external force is summed with tendon force, the Resultant force acting on the inertial load. Force feedback is represented without dynamic components, but an adjustable reflex delay is included because this was shown in the empiric work to stabilize positive force feedback. Feedback from muscle spindles is likewise represented without dynamics and without delays.

Many combinations of parameters were tested in simulations. Figure 2 shows a small set of simulations. All simulations were performed with the use of the Runge-Kutta-3 simulation algorithm in the Simulink program. Two variables are plotted: external force (perturbing input) and displacement (output).

\section{RESULTS}

Without displacement feedback or force feedback, the system, comprising the viscoelastic muscle parallel stiffness and the inertial load, showed a damped displacement response (Figure 2-1) to the external force.

Adding positive force feedback at loop gains produced stable load compensation (yield in Figure 2B with $G_{f}=0.5$ is about half that in Figure 2-1 without feedback).

In this model, $G_{f}$ was determined by inspection as the multiple of the static gains around the force loop. Because displacement is held constant for this purpose, the only elements involved are the muscle contractile element and the force feedback element. When $G_{f}=1$ in Figure 2B, apart from the small transients, the mass did not move, i.e., the system had infinite stiffness.

The loop remained stable up to $G_{f}=1.4$ (Figure 2C), exhibiting an affirming reaction (i.e., instead of yielding, the mass now moved in the opposite direction of the imposed force). The reason that the system remained stable for values of $G_{f}$ between 1 and 1.4 is that the parallel stiffness element provided negative displacement feedback and velocity feedback, which had a stabilizing effect (if the gain of this element was set to 0 , the system went unstable at $G_{f}=1$ ).
Adding a 25-ms delay to the force feedback pathway had a stabilizing effect (Figure 2D), allowing a higher loop gain to be attained with a larger affirming reaction (Figure 2F).

Note that the magnitude and sign of feedback gain are separately specified in this paper.

Negative force feedback in combination with negative displacement feedback resulted in a spring like response to external loading, the stiffness of which increased as $G_{d}$ increased and $G_{f}$ decreased (not illustrated). Comparison of Figures $3 \mathbf{E}$ and $3 \mathbf{H}$ shows another results when the displacement feedback gain is exponential instead of constant value. The stability of system increased and the settling time will become shorter so the system will be more rapid.

Figures 3F and 3G show that if we increase the force feedback to $G_{f}=2$, the amplitude of oscillation parts will increase and transient response of the system will become more oscillating and the settling time will also increase so increasing the $G_{f}$ for more than 1.4 will lead to oscillating the system, and it emphasize that max gain for obtaining optimum response is $G_{f}=1.4$. Figures $3 \mathbf{I}$ and $3 \mathbf{J}$ show that increasing the displacement feedback gain is effective in specific limitation. While $G_{d}=0.5$ in comparison of $G_{d}=1$ the system has more oscillating responses and the settling time is longer.

But in comparison of Figures 3I and 3J we could infer the optimum $G_{d}$ is approximately $G_{d}=1$, because increasing $G_{d}$ to value of 2 lead to oscillating the responses of the system. Although these oscillations are smaller than the oscillations of system when $G_{d}=0.5$ and the settling time is still shorter.

\section{DISCUSSIONS AND CONCLUSIONS}

In the simple reflex model the muscle contractile element is modeled as a first-order low-pass filter with a cutoff frequency of $8 \mathrm{~Hz}$, somewhat higher than the 

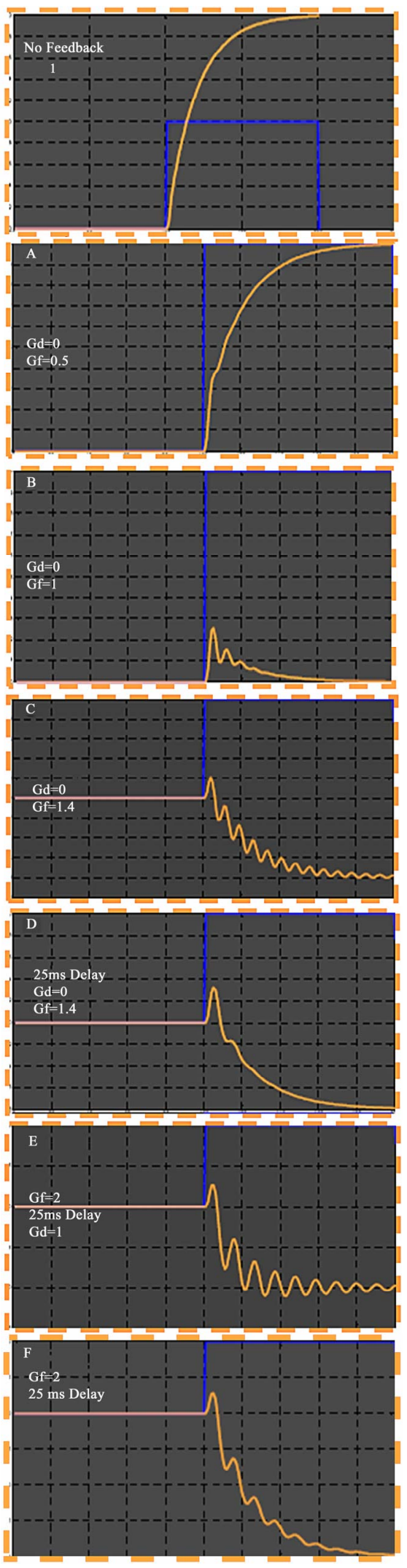

Figure 2. Simulations based on static reflex model of Figure 1.
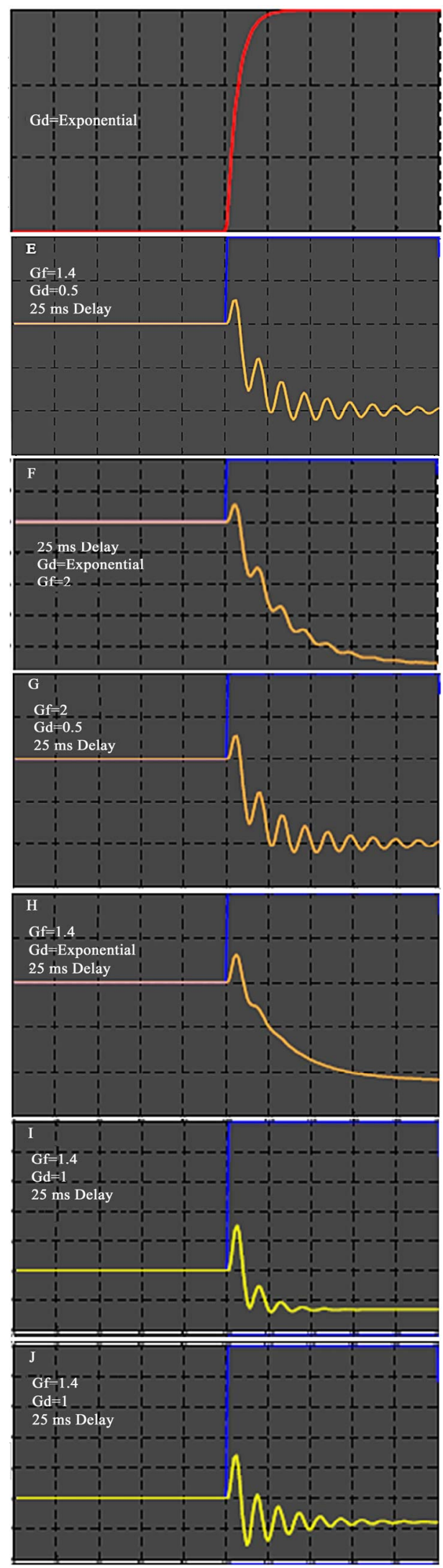

Figure 3. Exponential displacement feedback. 
isometric frequency response characteristic of cat triceps surae muscles of Rosenthal that positive force feedback may be appropriate in some et al. 1970. A mass of $1 \mathrm{~kg}$ represents the inertial load borne by a cat hind limb. A force increment of $10 \mathrm{~N}$ represents the mean force developed by triceps surae during the stance phase of slow gait [11]. Inherent muscle properties are simplified to a linear viscoelastic element with a stiffness of $0.5 \mathrm{~N} / \mathrm{mm}$. The force feedback and displacement feedback signals are represented without dynamics. It was clear that the reflex mechanism in question represented positive feedback and this was normally associated with instability. It was tacitly assumed that the nervous system would somehow always limit positive force feedback gain within a range consistent with stability. Our results suggest that a combination of intrinsic muscle properties, concomitant negative displacement feedback, and reflex delays found in neuromuscular may provide this automatic gain control.

Our experiments and analysis verify that positive force feedback in the neuromuscular system can provide stable and effective load compensation. The analysis also shows that the conclusions regarding the stabilizing influence of muscle intrinsic properties, length feedback, and delays in positive feedback pathways were robust in the face of large parametric and structural variations in the systems considered. Stable behavior for large values of positive feedback gains was unexpected and initially quite puzzling. However, it became apparent that loop gain did not remain high, but rather it was automatically attenuated when muscles shortened and thereby reduced their force producing capability.

\section{REFERENCES}

[1] Brownstone, R. M., Gossard, J. P., and Hultborn, H., (1994) Voltage-dependent excitation of motoneurones from spinal locomotor centres in the cat, Springer-Verlag, Exp. Brain Res., 102, 3444.

[2] Grey, M. J., Mazzaro, N., Nielsen, J. B., and Sinkjær, T., (2004) Ankle extensor proprioceptors contribute to the enhancement of the soleus EMG during the stance phase of human walking1, Can. J. Physiol. Pharmacol., 82, 610-616.

[3] Donelan, J. M. and Pearson, K. G., (2004) Contribution of sensory feedback to ongoing ankle extensor activity during the stance phase of walking, Can. J. Physiol. Pharmacol, 82, 589-598.

[4] Frigon, A. and Rossignol, S., (2006) Experiments and models of sensorimotor interactions during locomotion, Springer Berlin/Heidelberg, Saturday, DOI: 10.1007/s 00422-006-0129-x.

[5] Prochazka, A. and Gillard, D., (1997) Sensory control of locomotion, Proceeding of American Control Conference.

[6] Prochazka, A., Gillard, D., and Bennet, D. J., (1997) Implications of positive feedback in the control of movement, the American Physiological Society 0022-3077/97, 5.00 Copyright.

[7] Taylor, A., Ellaway, P. H., Durbaba, R., and Rawlinson, S., (2000) Distinctive patterns of static and dynamic gamma motor activity during locomotion in the decerebrate cat, Journal of Physiology, 529(3), pp. 825-836.

[8] Raya, J. G., Ramı́rez, A., and Muñoz-Martı́nez, E. J., (2004) Gamma 3 alpha linkage and persistent firing of Ia fibers by pudendal nerve stimulation in the decerebrate cat, J. Neurophysiol, 92, 387-394.

[9] Krakauer, J. W., MD, (2006) Spinal cord physiology: Receptors and reflexes.

[10] Csercsik, D., (2006) Construction of simple dynamic models of the gamma-loop mechanism, Research Report SCL-001.

[11] Knutson, G. A., Edward, D. C., and Owens, F., MS, DC, (2003) Active and passive characteristics of muscle tone and their relationship to models of subluxation, 00083194/2003/269-283/\$2.00/JCCA. 